\title{
Physical Fitness Differences between Freestyle and Greco-Roman Junior Wrestlers
}

\author{
by \\ Erkan Demirkan ${ }^{1}$, Mehmet Kutlu², Mitat Koz ${ }^{3}$, Mehmet Özal ${ }^{4}$, Mike Favre 5
}

\begin{abstract}
The aim of the present study was to examine physical fitness differences between Freestyle and Greco-Roman junior wrestlers. One hundred twenty-six junior wrestlers, comprising 70 Freestyle and 56 Greco-Roman wrestlers, participated in this study. The somatic and physical fitness profile included body mass, body height, body mass index, body composition, flexibility, maximal anaerobic power of the legs and arms, aerobic endurance, hand grip strength, leg and back strength, and speed. No significant differences were found in the anthropometric and physical features between Freestyle and Greco-Roman wrestlers. The Greco-Roman wrestlers had a significantly higher level of relative leg power, peak arm power, relative peak arm power, and relative average arm power than Freestyle wrestlers $(p<0.05)$. Greco-Roman wrestlers were significantly faster, had better agility, and had a greater level of leg strength than Freestyle wrestlers, but Freestyle wrestlers were more flexible than Greco-Roman wrestlers $(p<0.05)$. Discriminant function analysis indicated that peak arm power, agility, speed, and flexibility were selective factors for the differences between Freestyle and Greco-Roman wrestlers. In conclusion, the present study indicates that the differences between these wrestling styles promote physical fitness differences in elite wrestlers. The results reflect specific features of each wrestling style.
\end{abstract}

Key words: Anaerobic power, aerobic capacity, body composition, speed, strength, wrestlers.

\section{Introduction}

Wrestling is a sport discipline that places great demands on athletes in terms of physical preparation (Sterkowicz-Przybycień et al., 2011). Two wrestling styles, Freestyle and Greco-Roman, are included for men in the Olympics. Freestyle wrestling includes upper and lower body wrestling and is characteristic of short duration, high-intensity intermittent effort that lasts a total of $6 \mathrm{~min}$ for senior and junior wrestlers $(2 \times 3$-min bouts). Anaerobic power is crucial because of the scoring system for Freestyle wrestling, which uses explosive techniques that may end the match before regulation time (Cipriano, 1993; Mirzaei et al., 2009). Greco-Roman wrestling allows only upper body moves and also has a bout duration of 6 min $(2 \times 3$-min bouts $)$ for senior and junior wrestlers according to new official rules. The sports level clearly differentiates the results of tests of strength endurance of arm and trunk muscles, of which function is extremely important in wrestling (Sterkowicz and Starosta, 2005). The importance of lower and upper body power lies in the ability to lift the opponent during offensive maneuvers and resist attacks while in defense.

\footnotetext{
1 - Physical Education and Sports School, Hitit University, Çorum- TURKEY.

2 - Department of Physical Education and Sport, Education Faculty, the University of Kırıkkale, Kırıkkale- TURKEY.

3 - Sports Sciences Faculty, Ankara University, Ankara - TURKEY.

${ }^{4}$ Department of Sport - General Diretorate of Sport, Ankara - TURKEY.

5 - Director of Olympic Sports SEC, University of Michigan. USA
} 
Elite wrestlers have been characterized by high maximal power output of the arm and leg muscles (Yoon, 2002).

Only a few studies have compared the physical fitness characteristics of both wrestling styles (Horswill, 1992; Baić et al., 2007; Gullón et al., 2011). Therefore, the present study compared the level of physical fitness between Freestyle and Greco-Roman elite wrestlers. Our hypothesis was that the official rule differences between the Freestyle and Greco-Roman styles promote physical fitness differences based on technical implementation in wrestling, in which the implementation of the different styles of wrestling partially influences the physical fitness of wrestlers.

\section{Material and Methods}

\section{Subjects}

One hundred twenty-six junior competitive wrestlers, 56 Greco-Roman wrestlers (16.4 \pm 0.7 years old) and 70 Freestyle wrestlers $(16.5 \pm 0.6$ years old $)$, volunteered to participate in the study. The sample was composed of the best junior wrestlers in Turkey. All of the participants were invited to the national team camp. Before participating in the study, the parents of the subjects read and signed an informed consent form. The athletes were asked not to participate in daily training programs within $24 \mathrm{~h}$ prior to testing. Testing was completed for all of the wrestlers in the same laboratory and field facilities on three consecutive days. The subjects and coaches were informed about the experimental procedures and possible risks and benefits of the project. The study complied with the Declaration of Helsinki and was approved by the Bioethics Commission of the University of Ankara in Turkey.

\section{Body height and body mass}

Body height and body mass measurements were made using a digital scale (Seca 664, Hamburg, Germany) with the subjects barefoot and wearing only shorts.

\section{Body composition}

Body composition was determined by measuring the skinfold thickness on three parts of the body (subscapular, triceps, abdominal) with a Holtain caliper. Skinfolds were measured three times at each site to the nearest $0.5 \mathrm{~mm}$, and the mean value was recorded. All skinfold measurements were taken on the right side of the body. Body fat content was calculated from the formula developed by Lohman (6). Fat-free mass was calculated by subtracting the fat tissue mass (in $\mathrm{kg}$ ) from the total body mass.

\section{Anaerobic power and capacity}

Wingate (WAnT) tests were used for the arms and legs during separate sessions. The lower-limb Wingate test consisted of 30-s supramaximal cycling against a resistance load of $75 \mathrm{~g} \cdot \mathrm{kg}^{-1}$ of body mass. Each test was performed on a Monark cycle ergometer (Model 894-E). Verbal encouragement was given to motivate the participants during the test (Inbar et al., 1996).

Arm cranking was performed with a standing body posture using a Monark 894E ergometer. Resistance load of $55 \mathrm{~g} \cdot \mathrm{kg}^{-1}$ of body mass was used for the upper limbs. The total number of revolutions performed during the entire $30 \mathrm{~s}$ test was counted, and power was calculated using a computerized MCE system, version 4.5 (JBM, Poland; Inbar et al., 1996). Verbal encouragement was given to motivate the participants during the test. During both arm cranking and leg cycling, mean power was defined as the average power generated during the $30 \mathrm{~s}$ interval (Hübner-Woźniak et al., 2004).

\section{Sprint test}

After a standardized 15-min warm-up (low-intensity running, several acceleration runs, and stretching exercises), the subjects underwent a sprint test that consisted of two maximal $30 \mathrm{~m}$ sprints with timing at 10 and $30 \mathrm{~m}$, with a $3 \mathrm{~min}$ rest period between each sprint.

\section{Maximal hand grip and leg-back strength}

Each subject's grip strength was measured for each hand using a Dynamometer (Takei A5001 Hand Grip Dynamometer, Tokyo, Japan). The average of two trials was recorded. Maximal leg and back strength (BS) was measured using a back muscle dynamometer (Takei A5002 Back and Leg Dynamometer, Tokyo, Japan). The average of two trials was recorded.

\section{Flexibility}

Flexibility of the trunk was determined using a standard sit and reach test (Eveque, Sit and Reach bench, Cheshire, England). The recorded score for this test was the average of two trials. 


\section{Aerobic endurance}

Aerobic endurance was determined in a $20 \mathrm{~m}$ shuttle run test. The wrestlers started running back and forth on a $20 \mathrm{~m}$ course and touched the line at the end. The initial speed was $8.0 \mathrm{~km} / \mathrm{h}$, which was increased by $0.5 \mathrm{~km} / \mathrm{h}$ every minute, in accordance with a pace dictated by a sound signal on an audiotape. The wrestlers were instructed to keep pace with the signal for as long as possible. When the subjects could no longer follow the pace, the last stage recorded was used to predict $\mathrm{VO} 2 \mathrm{max}$. A predicted $\mathrm{VO} 2 \mathrm{max}$ was obtained using the equation of Leger and Gadoury (1989).

\section{Data analysis}

The general characteristics of the participants are presented as means and standard deviations (SD). The differences between the Freestyle and Greco-Roman wrestlers were determined using an independent $t$-test. Additionally, discriminant function analysis was performed to determine which set of variables most accurately predicted wrestling styles. The level of significance for all of the statistical analyses was $p<0.05$.

\section{Results}

The physical characteristics and training experience of the Freestyle and Greco-Roman wrestlers are presented in Table 1. No significant differences were found between the characteristic features of Freestyle and Greco-Roman wrestlers.

The arm and leg anaerobic power and capacity of the Freestyle and Greco-Roman wrestlers are presented in Table 2. The GrecoRoman wrestlers had significantly higher relative average leg power $(\mathrm{W} / \mathrm{kg})$, peak arm power $(\mathrm{W})$, relative peak arm power $(\mathrm{W} / \mathrm{kg})$, and relative average arm power $(\mathrm{W} / \mathrm{kg})$ than Freestyle wrestlers $(p<0.05$; Table 2$)$.

Aerobic endurance, speed, agility, strength, and flexibility in Freestyle and GrecoRoman wrestlers are presented in Table 3. The Greco-Roman wrestlers were significantly faster (10 m speed test) and more agile, had higher leg strength, and were more flexible than Freestyle wrestlers $(p<0.05$; Table 3$)$.

Discriminant analysis was used to test the differences between the Freestyle and GrecoRoman elite adolescent wrestlers with regard to physical fitness variables. Five variables were put into the classification function of the discriminant function procedure: peak arm power, agility, speed (both 10 and $30 \mathrm{~m}$ ), and flexibility. These variables resulted in a final Wilks' lambda of 0.386 $(p<0.05)$ and an average squared canonical correlation of 0.78 , indicating that these variables accounted for approximately $61 \%(0.784)$ of the variance in predicting group membership. This model correctly classified 49 of 56 subjects for Greco-Roman wrestlers and 61 of 70 subjects for Freestyle wrestlers, with an overall prediction accuracy of $87.5 \%$ (Table 4 ).

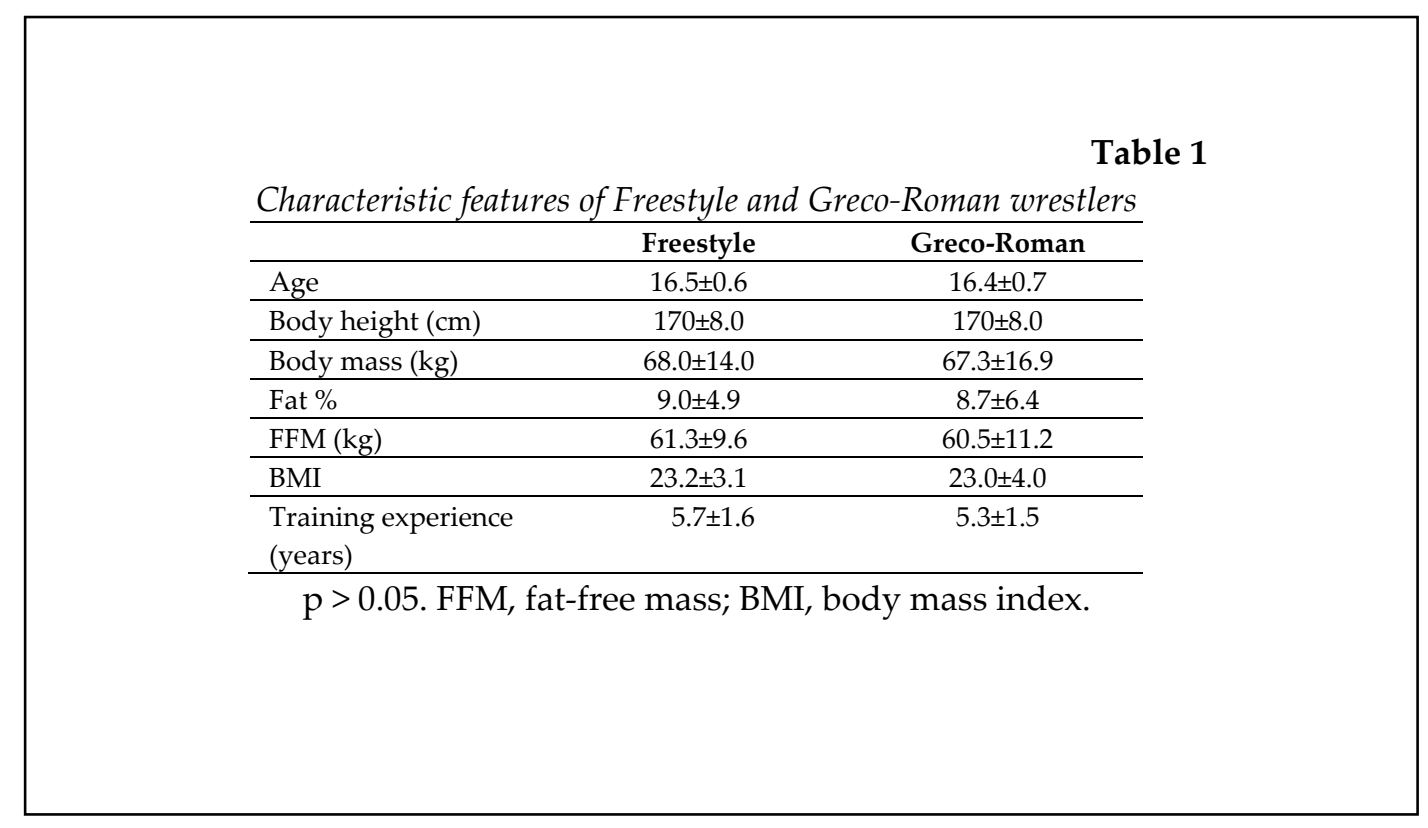


Table 2

Comparison of anaerobic upper and lower limb power and capacity

\begin{tabular}{lccc}
\hline Variables & Freestyle & Greco-Roman & $p$ \\
\hline Peak leg power $(\mathrm{W})$ & $895 \pm 210$ & $906 \pm 250$ & 0.77 \\
\hline Relative peak leg power $(\mathrm{W} / \mathrm{kg})$ & $13.2 \pm 2.0$ & $13.5 \pm 1.6$ & 0.43 \\
\hline Average leg power $(\mathrm{W})$ & $461 \pm 100$ & $478 \pm 119$ & 0.39 \\
\hline Relative average leg power $(\mathrm{W} / \mathrm{kg})$ & $6.8 \pm 0.8$ & $7.1 \pm 0.6$ & 0.02 \\
\hline Peak arm power $(\mathrm{W})$ & $594 \pm 173$ & $693 \pm 218$ & 0.01 \\
\hline Relative peak arm power $(\mathrm{W} / \mathrm{kg})$ & $8.7 \pm 2.0$ & $10.2 \pm 1.8$ & 0.00 \\
\hline Average arm power $(\mathrm{W})$ & $316 \pm 94$ & $348 \pm 96$ & 0.06 \\
\hline Relative average arm power $(\mathrm{W} / \mathrm{kg})$ & $4.6 \pm 0.9$ & $5.1 \pm 0.6$ & 0.00 \\
\hline
\end{tabular}

$\mathrm{p}<0.05$. W: watt.

Table 3

Comparison of aerobic endurance, speed, agility, strength, and flexibility

\begin{tabular}{lccc}
\hline Variables & Freestyle & Greco-Roman & $p$ \\
\hline VO2max $\left(\mathrm{ml} \cdot \mathrm{kg} \cdot \mathrm{min}^{-1}\right)$ & $50.1 \pm 6.3$ & $51 \pm 4.9$ & 0.42 \\
\hline $10 \mathrm{~m}$ speed $(\mathrm{s})$ & $1.85 \pm 0.1$ & $1.74 \pm 0.1$ & 0.00 \\
\hline 30 m speed $(\mathrm{s})$ & $4.30 \pm 0.3$ & $4.39 \pm 0.2$ & 0.05 \\
\hline Agility $(\mathrm{s})$ & $15.4 \pm 0.8$ & $14.6 \pm 0.6$ & 0.00 \\
\hline Right hand strength $(\mathrm{kg})$ & $43.9 \pm 9.1$ & $45.7 \pm 9.3$ & 0.28 \\
\hline Left hand strength $(\mathrm{kg})$ & $43.4 \pm 8.8$ & $44.6 \pm 9.0$ & 0.47 \\
\hline Back strength $(\mathrm{kg})$ & $148 \pm 39$ & $154 \pm 26$ & 0.37 \\
\hline Leg strength $(\mathrm{kg})$ & $180 \pm 40$ & $204 \pm 32$ & 0.00 \\
\hline Flexibility $(\mathrm{cm})$ & $34 \pm 7.0$ & $30 \pm 6.2$ & 0.01 \\
\hline & $p<0.05$. & &
\end{tabular}

Table 4

Standardized canonical discriminant function coefficients

\begin{tabular}{lccc}
\hline Variables & $\begin{array}{c}\text { Function } \\
\text { coefficients }\end{array}$ & Freestyle & $\begin{array}{c}\text { Greco- } \\
\text { Roman }\end{array}$ \\
\hline Peak arm power $(\mathrm{W} / \mathrm{kg})$ & -0.534 & $8.7 \pm 2.0$ & $10.2 \pm 1.8$ \\
\hline Agility $(\mathrm{s})$ & 0.684 & $15.4 \pm 0.8$ & $14.6 \pm 0.6$ \\
\hline 10 m speed $(\mathrm{s})$ & 0.705 & $1.85 \pm 0.1$ & $1.74 \pm 0.1$ \\
\hline 30 m speed $(\mathrm{s})$ & -1.163 & $4.30 \pm 0.3$ & $4.39 \pm 0.2$ \\
\hline Flexibility $(\mathrm{cm})$ & 0.322 & $34 \pm 7.0$ & $30 \pm 6.2$ \\
\hline
\end{tabular}




\section{Discussion}

The primary findings of this investigation indicated that elite level Greco-Roman and Freestyle junior wrestlers presented similar training backgrounds, body composition, and anthropometrical characteristics (Table 1). These results are consistent with previous studies that reported no differences in any of the anthropometrical and physical characteristics between the Freestyle and Greco-Roman groups (Horswill, 1992; Demirkan et al., 2011; Gullón et al., 2011). However, the present results confirmed a statistically significant difference in anaerobic arm power and capacity between the GrecoRoman and Freestyle wrestlers, but anaerobic leg power (with the exception of average relative leg power) and capacity were similar (Table 2). These results also showed that top-level Greco-Roman wrestlers had a higher level of anaerobic power and capacity $(14.3 \%$ peak arm power $[\mathrm{W}], 14.7 \%$ relative peak arm power, and $9.8 \%$ relative average peak arm power) in the upper extremities than Freestyle wrestlers. These changes were most likely related to the fact that the competitors performed dynamic moves during both training and wrestling combat (i.e., lifting, throwing, and resisting opponents, which require upper body power), and all of the techniques in Greco-Roman wrestling must be performed with the upper body. Consequently, according to our results, we may conclude that good physical preparation of the upper extremities in Greco-Roman wrestlers is not only important but also a result of the longterm drilling of technical-tactical elements during the training process.

Scarce data are available on the physiological differences among elite GrecoRoman and Freestyle wrestlers. Horswill et al. (1992) found no significant differences in the mean and peak lower and upper limb power attained during a $30 \mathrm{~s}$ Wingate test between wrestlers of both styles. Gullon et al. (2011) found that no significant differences in the crank-arm Wingate test, either absolute or normalized to fatfree mass, between Freestyle and Greco-Roman wrestlers. This may be attributable to the grouping of variables (i.e., age, elite-nonelite, and sport experience). However, this investigation found no significant differences in aerobic endurance between Freestyle and Greco-Roman wrestlers. These results appear to be consistent with previous studies (Horswill, 1992; Gullón et al., 2011). Aerobic performance may be a basic requirement for wrestlers because a high level of aerobic power allows the athlete to maintain a high intensity of activities during a match and provides effective recovery during the $30 \mathrm{~s}$ rest period between the two 3-min rounds.

Significant differences in speed, agility, and flexibility were detected between the wrestling styles (Table 3). The statistical analysis indicated that Greco-Roman wrestlers were faster $(6.3 \%)$ and more agile (5.5\%) than Freestyle wrestlers. However, Freestyle wrestlers were more flexible $(11.8 \%)$ than Greco-Roman wrestlers. Additionally, the discriminant function analysis revealed that the significantly different variables were peak arm power $(\mathrm{W} / \mathrm{kg})$, agility, speed $(10$ and $30 \mathrm{~m})$, and flexibility among both wrestling styles (Table 4). According to these results, Greco-Roman wrestlers had higher peak arm power $(693 \pm 218 \mathrm{~W})$, were faster in $10 \mathrm{~m}(1.74$ $\pm 0.1 \mathrm{~s})$, and were more agile $(14.6 \pm 0.6 \mathrm{~s})$ than Freestyle wrestlers $(594 \pm 173 \mathrm{~W}, 1.85 \pm 0.1 \mathrm{~s}$, and $15.4 \pm 0.8 \mathrm{~s}$, respectively), but Freestyle wrestlers were more flexible $(34 \pm 7.0 \mathrm{~cm})$ and faster in $30 \mathrm{~m}$ $(4.30 \pm 0.3 \mathrm{~s})$ than Greco-Roman wrestlers $(30 \pm 6.2$ $\mathrm{cm}$ and $4.39 \pm 0.2 \mathrm{~s}$, respectively). In contrast to these results, Baic et al. (2007) indicated that toplevel Freestyle wrestlers had a higher level of strength endurance of the trunk and upper extremities than Greco-Roman wrestlers, based on discriminant function analysis. These authors assumed that their results were influenced by the specific features of each wrestling style. In another study, Gullon et al. (2011) compared both wrestling styles and found no differences in $10 \mathrm{~m}$ sprint times.

Numerous studies have been conducted to investigate differences between successful and less successful wrestlers and between male and female wrestlers (Roemmich and Frappier, 1993; Hübner-Woźniak et al., 2004; Vardar et al., 2007; Abellán et al., 2010; Pallares et al., 2011; Pallares et al., 2012). In one of these studies, Roemmich and Frappier (1993) employed discriminate function analysis to determine which collection of variables most accurately predicted wrestling success. They found that grip strength of the left hand, flexibility of the lower back and hamstrings, pushups, strength of the right quadriceps, and total distance covered during a 12-min run were 
important in predicting wrestling success. In another study, Palleres et al. (2011) used binary logistic regression analysis to predict the probability of being an elite wrestler. They found that training experience, fat-free mass, one repetition maximum (1RM) strength and muscle power in the bench press and full squat, and peak power were selective factors. Palleres et al. (2012) used regression analysis and found that fat-free mass and 1RM strength were the most significant factors of successful female wrestling performance.

Superior upper body strength and anaerobic capacity in Greco-Roman wrestling might be more beneficial for the initiation of attacks and explosive execution of wrestling techniques because only upper body moves are allowed. This may stimulate the development of anaerobic power and capacity of the upper body. However, Freestyle and Greco-Roman wrestlers had similar lower body anaerobic power and capacity. Freestyle is a complex wrestling style that allows actions of both the upper and lower parts of the body. It requires the strength and power of both body parts. These results indicate that the increasing of upper body power requires also the developed lower body power.

\section{Conclusion}

Freestyle and Greco-Roman wrestlers have similar characteristic features (age, body height, body mass, fat percentage, fat-free mass, and body mass index) and sports experience, but Greco-Roman wrestlers have a higher level of anaerobic upper body power and capacity than Freestyle wrestlers. Greco-Roman wrestlers perform dynamic moves (e.g., lifting, throwing, and resisting opponents) that require upper body power, and all of the techniques in Greco-Roman wrestling must be performed with the upper body, both in competitions and training.

\section{References}

Abellán AM, Pallarés JG, Gullón JML, Otegui XM, Baños VM, Moreno AM. Anaerobic Factors to Predict Wrestling Performance. Cuadernos De Psicología Del Deporte, 2010; 10: 17-23

Baić M, Sertić H, Starosta W. Differences in Physical Fitness Levels Between The Classical and The Free Style Wrestlers. Kinesiology, 2007; 2:142-149

Cipriano NA. Technical-tactical analysis of freestyle wresling. J Strength Cond Res, 1993; 7: 133-140

Demirkan E, Kutlu M, Koz M. The Segmental Body Composition Comparison of Freestyle and Greco-Roman Style Wrestlers with Bioelectrical Impedance Method. Selçuk University The Journal of Physical Education and Sports Science, 2011; 13 (3): 340-343

Gullón JML, Xabier M, Dolores M, Torres-Bonete MI, Pallarés JG. Physical fitness differences between Freestyle and Greco-Roman elite wrestlers. Arch Budo, 2011; 7(4): OA217-225

Heyward VH, Stolarczyk LM. Applied body composition assessment. Human Kinetics; 1996

Hübner-Woźniak E, Kosmol A, Lutoslawska G, Bem EZ. Anaerobic performance of arms and legs in male and female free style wrestlers. J Sci Med Sport, 2004; 7(4): 473-80

Horswill CA. Applied Physiology of Amateur Wrestling. Sport Med,1992; 14: 114-143

Inbar O, Bar-Or O, Skinner JS. The Wingate Anaerobic Test. Champaign. Human Kinetics; 1996

Léger L, Gadoury C. Validity of the $20 \mathrm{~m}$ shuttle run test with 1 min stages to predict VO2max in adults. Can J Sport Sci, 1989; 14(1): 21-6

Mirzaei B, Curby DG, Rahmani-Nia F, Moghadasi M. Physiological profile of elite Iranian junior freestyle wrestlers. J Strength Cond Res, 2009; 23(8): 2339-2344

Roemmich JN, Frappier JP. Physiological Determinants of Wrestling Success in High School Athletes. Pediatric Exercise Science, 1993; 5: 134-144

Pallares JG, Lopez JM, Muriel GX, Mikel Izquierdo AD. Physical Fitness Factors to Predict Male Olympic 
Wrestling Performance. Eur J Appl Physiol, 2011; 111: 1747-1758

Pallarés JG, López-Gullón JM, Torres-Bonete MD, Izquierdo M. Physical Fitness Factors to Predict Female Olympic Wrestling Performance and Sex Differences. J Strength Cond Res, 2012; 26(3): 794-803

Sterkowicz S, Starosta W. Selected factors influencing the level of general fitness in elite Greco Roman wrestlers. JHK, 2005; 14: 93-104

Sterkowicz-Przybycień KL, Sterkowicz S, Zarów RT. Somatotype, Body Composition and Proportionality in Polish Top Greco-Roman Wrestlers. JHK, 2011; 28: 141-154

Vardar SA, Tezel S, Öztürk L, Kaya O. The relationship between body composition and anaerobic performance of elite young wrestlers. J Sports Sci Med, 2007; 6(CSSI-2): 34-38

Yoon J. Physiological Profiles of Elite Senior Wrestlers. Sports Med, 2002; 32 (4): 225-233

\section{Corresponding author:}

Erkan Demirkan (PhD

Physical Education and Sports School, Hitit University, Çorum- TURKEY

Phone: +90 3642277923

Fax: +903642277921

E-mails: erkandemirkan_1979@hotmail.com, erkandemirkan@hitit.edu.tr 